\title{
Visualization of uncertain scalar data fields using color scales and perceptually adapted noise
}

\author{
Alexandre Coninx* \\ LPPA - CNRS - Collège de France \\ LJK - Grenoble University - INRIA \\ EDF R\&D
}

\author{
Georges-Pierre Bonneau ${ }^{\dagger}$ \\ LJK - Grenoble University - INRIA \\ Guillaume Thibault ${ }^{\S}$ \\ EDF R\&D \\ LPPA - CNRS - Collège de France
}

\author{
Jacques Droulez $z^{\ddagger}$ \\ LPPA - CNRS - Collège de France
}

\begin{abstract}
We present a new method to visualize uncertain scalar data fields by combining color scale visualization techniques with animated, perceptually adapted Perlin noise. The parameters of the Perlin noise are controlled by the uncertainty information to produce animated patterns showing local data value and quality. In order to precisely control the perception of the noise patterns, we perform a psychophysical evaluation of contrast sensitivity thresholds for a set of Perlin noise stimuli. We validate and extend this evaluation using an existing computational model. This allows us to predict the perception of the uncertainty noise patterns for arbitrary choices of parameters. We demonstrate and discuss the efficiency and the benefits of our method with various settings, color maps and data sets.
\end{abstract}

CR Categories: I.3.3 [Computer Graphics]: Picture/Image Generation -Display algorithms; I.3.8 [Computer Graphics]: Applications

Keywords: scientific visualization, uncertainty visualization, computer graphics, Perlin noise, psychophysics, contrast sensitivity

\section{Introduction}

As measurement tools, numerical models and computational capabilities progress and evolve, scientific datasets become increasingly large and complex, often related to multiple interdependent phenomenons with multiple spatial and temporal scales, and described by different physical quantities. Visual exploration of this data is now a required step in order to understand the underlying physical processes. It is also one of the premium means to communicate this knowledge by using static or animated images.

Uncertainty is always present in scientific data, and visualizing it efficiently has been acknowledged as one of the main challenges of visualization research. Uncertainty differs from other types of data first because it is a very general term which can cover many different concepts [Skeels et al. 2009], and second because uncertain data

\footnotetext{
*e-mail: alexandre.coninx@college-de-france.f

†e-mail: georges-pierre.bonneau@inria.fr

‡e-mail: jacques.droulez@college-de-france.fr

e-mail: guillaume.thibault@edf.fr
}

analysis is an implicitly multidimensional problem : from uncertain data we compute statistical parameters (like min and max, or mean and standard deviation) which form an at least bidimensional space that should be analyzed and displayed as a whole.

In this work, we propose a set of new results and techniques to visualize uncertain scalar fields in an accurate manner. More precisely we make the three following contributions:

- we propose a new method based on Perlin noise in conjunction with colormaps in order to visualize in a combined manner the data and its uncertainty,

- we perform a psychophysical evaluation of contrast sensitivity thresholds of Perlin noise,

- we validate and extend the results of the perceptive evaluation for arbitrary choices of the noise parameters using an existing computational model, in order to accurately control when and where the uncertainty is made visible.

The paper is structured as follows. In section 2, we describe the algorithmic machinery of our method, leaving apart perceptual issues. We point out at the end of this section the need to precisely assess the visibility of the noise patterns. In section 3 we describe the psychophysical experiment that has been conducted to measure contrast sensitivity thresholds for a set of given Perlin noise stimuli. Furthermore we show that the results of this experiment are in complete accordance with an existing computational model. This section has an interest per se, it could be used in other research works where a perception analysis of Perlin noise patterns is needed. In section 4 we combine the results of the two previous sections in order to come up with a visualization method for uncertain scalar data field. We show how the perceptual results of section 3 enable to control the parameters of the Perlin noise used for the colormap perturbation, in order to either ensure the visibility of the uncertainty for some values, or on the contrary hide the uncertainty for other values. We demonstrate and discuss the benefits of our method with various settings, colormaps and data sets. We conclude and give some possible future work in section 5 .

\section{Visualization of uncertain data using Perlin noise}

\subsection{Previous work}

\subsubsection{Visualization of uncertainty data}

Research on uncertainty visualization began with works from Wittenbrink and Pang, which were mostly targeted on uncertain vector data in a 2D field [Wittenbrink et al. 1996] and on positional uncertainty of 3D surfaces [Pang et al. 1997]. Further work proposed uncertainty visualization techniques for a variety of other 


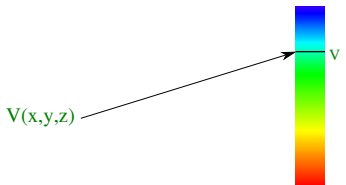

(a) Classical color scale visualization

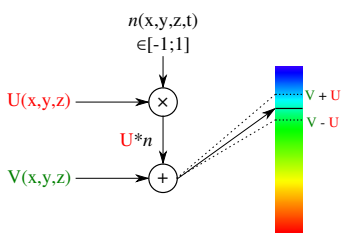

(c) Our method : the color scale lookup is biased by a Perlin noise weighted by uncertainty data

Figure 1: Comparison between classical visualization using color maps and our technique.

data types such as distribution data [Kao et al. 2005], isosurfaces and streamlines [Luo et al. 2003]. Despite some effort to classify uncertainty visualization techniques and give them a theoretical framework [Riveiro 2007; Griethe and Schumann 2006], much of this research adresses either specific datasets such as astrophysical data [Li et al. 2007] or medical data [Lundström et al. 2007] or specific visualization techniques like volume rendering [Djurcilov et al. 2002].

In this study, we focus on scalar fields, which are both extremely common and simple to visualize (without considering uncertainty) using color scales. A color scale provides an unidimensional mapping between the values of a scalar field and colors to be displayed on the screen. It is an important goal for uncertainty visualization to preserve color scales' ease of use and efficiency. Several proposals have been made to handle this problem by using bidimensional [Djurcilov et al. 2002] or even tridimensional [Luo et al. 2003] color maps, mapping both the mean value and one or more higher order statistical parameters about uncertainty (like standard deviation and kurtosis) to color. These methods succeed in jointly displaying data and uncertainty information, but at the cost of the readability and simplicity of use : 2D color maps lack the intuitive data order present in 1D color maps, which allows to quickly compare values and understand structures and spatial features in the data.

Other proposals focus on the idea of overlaying the classic color map visualization with additional visual items depicting uncertainty, such as gratings ([Cedilnik and Rheingans 2000]) and other repetitive patterns, or glyphs [Viard 2010]. Parameters such as glyph size, glyph density or line contrast can then be modulated by the uncertainty information. These methods give interesting results for visualization of $2 \mathrm{D}$ data, but are difficult to adapt to $3 \mathrm{D}$ spaces, where they tend to quickly overload the visualization and produce visual clutter. Methods using noise or textures to show uncertainty [Djurcilov et al. 2002] seem slightly more efficient for $3 \mathrm{D}$ datasets, but were only tested with direct volume rendering and could quickly mask the underlying visualization by color mapped

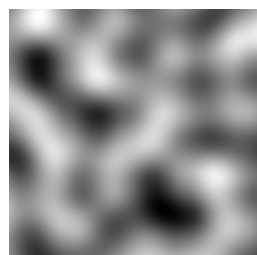

(a) $f_{0}=2 ; p=0$

(d) $f_{0}=4 ; p=0$

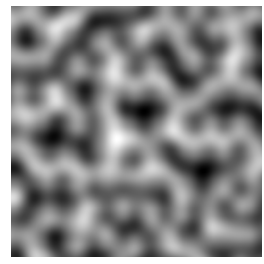

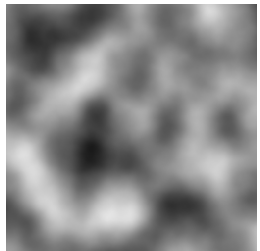

(b) $f_{0}=2 ; p=0.25$

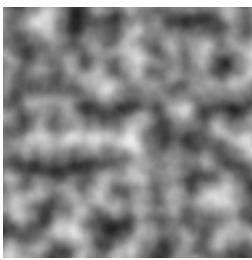

(e) $f_{0}=4 ; p=0.25$

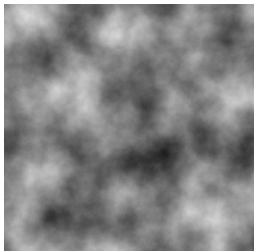

(c) $f_{0}=2 ; p=0.5$

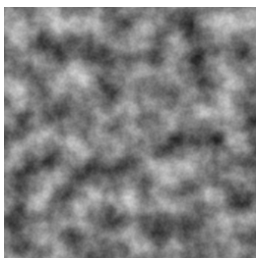

(f) $f_{0}=4 ; p=0.5$
Figure 2: Bidimensional Perlin noise for a few $f_{0}$ and $p$ parameters.

data.

\subsubsection{Perlin noise}

We chose to use Perlin noise [Perlin 1985] in this work because of its computational speed, existing parallel implementation, and ease of control of the visual and spectral features by two scalar parameters ( $f_{0}$ and $p$, explained below). We refer the reader to the excellent STAR on Procedural Noise Function [Lagae et al. 2010] for other possible choices. This section assumes the reader to be familiar with Perlin noise, and focuses on the notations.

In this work, we will use 4D Perlin noise, with three spatial dimensions that can be written as a position vector $\vec{x}$ and a time dimension $t$. The algorithm produces a four-dimensional approximately bandpass, isotropic noise, an animated solid texture $N(\vec{x}, t)$ with a spatial and temporal frequency content limited to a band around a central value determined by the lattice step size. The spatial frequency can be arbitrarily chosen simply by scaling the texture using an $f$ parameter : $N(f \vec{x}, t)$. The same can be done for the time dimension if desired. Spectral control of the noise can then be achieved by adding together several successive bands (or octaves) of noise with a weighted sum :

$$
\sum_{i} w_{i} N\left(f_{0} 2^{i} \vec{x}, t\right)
$$

where $N$ is our basic noise function, $\vec{x}$ the spatial coordinates, $f_{0}$ the fundamental frequency (the scaling parameter of the lowest frequency octave) and $w_{i}$ are the octave weights.

We define the weights as :

$$
w_{i}=p^{i}
$$

with $p \in[0 ; 1]$ being a persistence parameter controlling the spread of the noise. $p=0$ gives mono-octave noise, $p=1$ gives a result close to white noise, and intermediate values generate multi-octave noises where spectral power decreases with increasing frequency.

These parameters allow us to define a family of procedural textures as the following functions : 


$$
n_{f_{0}, p}(\vec{x}, t)=\sum_{i} p^{i} N\left(f_{0} 2^{i} \vec{x}, t\right)
$$

These functions will be used in conjunction with color maps to build our uncertain data visualization technique.

\subsection{Visualization technique description}

\subsubsection{Algorithmic principle}

Our goal is to propose a generic method which provides a way to visualize both a scalar data and a scalar uncertainty information (like error or standard deviation) on a complex 3D geometry, while abiding by the following constraints :

- The method should build onto classical 1D color maps, and the resulting visualization should be immediately understandable by anyone familiar with color maps ;

- The uncertainty data should not mask the basic data, or make its visualization misleading, more complex, less accurate or more difficult to interpret.

In a classical 3D color scale visualization, the value $V(\vec{x})$ of the data is linearly mapped to a color map coordinate, and then to the corresponding RGB color value, which is then displayed in the $3 \mathrm{D}$ visualization (Figure 1a). In our method, we want to modulate this visualization using an uncertainty value $U(\vec{x})$. For this, we use the 4D Perlin noise $n_{f_{0}, p}(\vec{x}, t)$ described in 2.1.2 and linearly scale its values so it has a null mean and takes values in $[-1 ; 1]$. We then multiply the scaled noise by the uncertainty value, and add the result to the $V$ base value. The result is a time-varying value $V(\vec{x})+n_{f_{0}, p}(\vec{x}, t) \cdot U(\vec{x})$. It takes values in $[V(\vec{x})-U(\vec{x}) ; V(\vec{x})+U(\vec{x})]$, has a mean of $V(\vec{x})$, and is used as a color map coordinate (Figure 1c, 1d).

\subsubsection{Implementation}

The Perlin noise algorithm has already been implemented on GPU in previous works [Gustavson 2005]. The whole technique was implemented on GPU using GLSL and tested in a custom demonstration program written in $\mathrm{C}++$.

\subsection{Discussion}

Our technique effectively allows to take into account uncertainty information in the visualization without overloading it with new visual items. The use of classic $1 \mathrm{D}$ colormaps allows the user to quickly visualize the shape of the scalar field and read numerical values, and the animated features introduced by Perlin noise efficiently conveys the concept of uncertain and unreliable information.

A consequence of our method is that the uncertainty information is only perceived in the areas where the patterns introduced by Perlin noise are salient enough to be detected by the human visual system. A way to take advantage of this behavior is to modify our technique to use a custom transfer function $g$ on the uncertainty value before multiplying it with the noise value : $V(\vec{x})+n_{f_{0}, p}(\vec{x}, t) \cdot g(U(\vec{x}))$. The $g$ function can be linear $(g(x)=G \cdot x ;$ Fig. 3e), in which case it introduces a simple gain factor $G$, allowing us to globally make the uncertainty less or more visible.

However, a high $G$ value tends to make the Perlin noise too salient for high uncertainty values and disrupts the visualization in the affected areas. We can therefore use non-linear transfert function, like the piecewise linear function shown in Fig. 3f, which allows us to

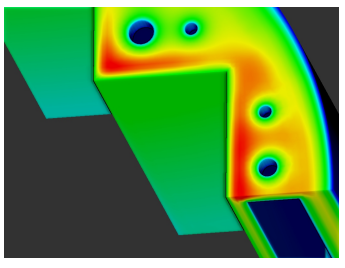

(a)

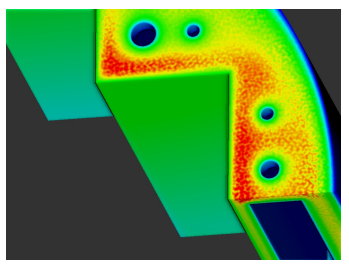

(c)

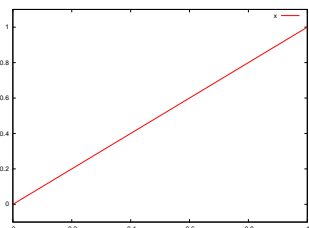

(e)

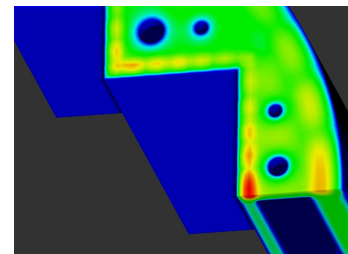

(b)

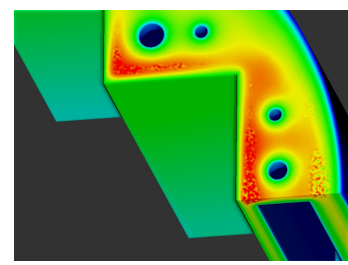

(d)

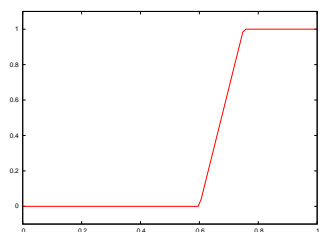

(f)
Figure 3: Examples of visualization of an uncertain scalar field using various techniques. We analyze the results of a $3 D$ thermics Monte Carlo simulation with 3000 runs. We visualize the mean temperature field (Fig. $3 a$ ) as data and the temperature standard deviation field (Fig. $3 b$ ) as uncertainty information. We can use either a linear transfer function (Fig. 3e) to visualize both the temperature field and the general shape of the uncertainty field,(Fig. 3c) or a threshold function (Fig. 3f) to show some specific features such as highly uncertain areas (Fig. $3 d$ ).

highlight some specific features of the uncertainty field while controlling the Perlin noise patterns visibility in the whole data field.

In order to put to best use these proposals and to be able to determine when the uncertainty information will and will not be perceived when using our method, it turns out to be necessary to study how the visual patterns generated by Perlin noise are processed by the human visual system and under which conditions (especially the $f_{0}$ and $p$ parameters values) they can be detected. We therefore conducted a perceptive evaluation of Perlin noise contrast sensitivity, which will be presented in the next section.

\section{Evaluation of luminance contrast sensitiv- ity of Perlin Noise}

\subsection{Previous work}

\subsubsection{Contrast sensitivity}

Luminance contrast sensitivity refers to the ability of the visual system to distinguish an object defined by a luminance pattern from a background. Human contrast sensitivity varies widely with stimuli, some visual patterns requiring a much higher luminance difference than others to be reliably perceived by the visual system. This variability has been studied for factors like spatial frequency 
[Kelly 1979b], temporal frequency [Kelly 1979a], features orientation [Campbell et al. 1966], retinal eccentricity [Robson and Graham 1981], overall luminance level (scotopic versus photopic vision) and stimulus hue [Mullen 1985]. Human contrast sensitivity also differs significantly between individuals, and evolves with aging.

The study of the variations of contrast sensitivity with the spatial frequency power spectrum has led to the concept of contrast sensitivity function, which describes the relationship between the spatial frequency of a carefully chosen stimulus (usually a sine wave grating or Gabor patch) and the contrast sensitivity of the observers [Campbell and Robson 1968]. Further work led to the idea of multiple frequency channels in the human visual system [Blakemore and Campbell 1969]. These findings were used both in the clinical field, where they were used to develop new diagnosis tools for ophthalmology [Owsley 2003], and for modelisation purposes, where they allowed the development of contrast sensitivity models of human vision [Watson 2000].

Human contrast sensitivity function measurements show a maximum around 4 to 5 cycles per degree, with sensitivity decreasing for lower and higher spatial frequencies. The sensitivity values vary between subjects and with the other previously discussed factors, but the shape of the curve and the maximum location remains consistent. Since Perlin noise's parameters directly control its spatial power spectrum, we should expect the same overall behavior for Perlin noise stimuli.

However, we can't simply rely on previous studies to assess the contrast sensitivity for Perlin noise. These studies mostly make use of sine wave gratings and Gabor patches, which are sine wave gratings limited by a gaussian envelope. These patterns are completely nonrandom and are extremely anisotropic, which results in a very simple phase spectrum and concentration of all of the contrast energy along the axis of the sine wave variation. In the opposite, Perlin noise is a random procedural texture and is mostly isotropic, with a random phase spectrum and contrast energy spread between all directions. Current research suggests that while contrast sensitivity functions accurately describe response to Gabor patterns, they're not so useful to explain sensitivity to other stimuli. Notably, models based on contrast sensitivity filters, often give higher errors for more complex stimuli like binary noise or natural images [Watson and Ahumada 2005].

\subsubsection{Analysis of Perlin noise}

To the best of our knowledge, no psychophysical work on the perceptual characteristics of Perlin noise has been conducted previously. Some research has been conducted on the spectral and mathematical characteristics of noise textures. Lagae et al. reviewed 6 different procedural noises, including Perlin noise [Lagae et al. 2010]. They find that all of them have quite close power spectrums : approximately band-pass, and (except for wavelet noise) isotropic.

\subsection{Experiment: contrast sensitivity thresholds for Perlin noise}

We performed a psychophysical experiment to assess contrast sensitivity thresholds for Perlin noise stimuli with different $f_{0}$ and $p$ parameters. Such contrast sensitivity measurement have already been done in previous works for various other stimuli.

One of these work is the ModelFest database [Carney et al. 1999], an effort from numerous vision scientists to build a contrast sensitivity threshold database for 43 different stimuli, both simple (gabor or gaussian patches) and complex (multiple gabor patches, natural image, ...) The related articles [Carney et al. 1999; Watson 2000] and the project web site [Watson 1999] define a standard methodology to measure sensitivity values for any luminance stimuli.

We decided to follow this method for our experiment. It allows us to use an already tested and validated methodology, and to directly compare our results to those obtained for other types of stimuli. Besides, the ModelFest data has been used to propose computational models of spatial contrast detection [Watson 2000; Watson and Ahumada 2005]. We can take advantage of these models to validate the collected data and extend our work.

\subsubsection{Experiment description}

Perlin noise luminance stimuli We chose 4 values of each parameter to perform our experiment : $f_{0} \in\{2,4,8,16\} \mathrm{cpd}$ and $p \in\{0,0.25,0.5,0.75\}$. We therefore measured contrast sensitivity for 16 points $\left(f_{0}, p\right)$ of the Perlin noise parameter space.

Following the ModelFest methods, stimuli consisted of 256x256 pixels luminance images, which had to be viewed by the subjects with a 120 pixels per degree resolution. In order to generate these stimuli, we wrote a Python program implementing Perlin's algorithm as described on his web site [Perlin 1999], except it uses the fifth-order interpolant used in his further work [Perlin 2002]. We used this program to generate $256 \times 256$ arrays containing bidimensional Perlin noise with the required $f_{0}$ and $p$ values. For $p>0$ we used a multiple octave weighted summation as described in section 2.1.2. The data for each noise array was centered and rescaled so the values are in $[-1 ; 1]$ and have a null mean. Each array was then multiplied by a gaussian mask with $\sigma=0.05^{\circ}$, like most of the stimuli from the ModelFest database.

In order to generate luminance images with a variable contrast from these arrays, to each pixel $n$ in the noise array we apply the following formula (adapted from [Watson and Ahumada 2005]) :

$$
L(n)=L_{0}(1+c * n)
$$

where $L_{0}$ is the mean luminance $\left(30.0 \mathrm{~cd} / \mathrm{m}^{2}\right.$ from ModelFest methods) and $c$ the desired Michelson contrast [Michelson 1962].

The resulting luminance stimuli are then used within the experimental setup described in the next section. Note that since Perlin noise is not a deterministic function, each stimulus is different and has a random aspect, although they have controlled $f_{0}, p, c$ and $L_{0}$ values. Preliminary tests and the fact that our psychometric method converge despite this randomness shows that it doesn't prevent efficient threshold determination.

Material and methods We again abided by the requirements of the ModelFest methods [Watson 1999]. We presented the stimuli during a 2IFC psychophysical task. For each $\left(f_{0}, p\right)$ values we used the Psi method [Kontsevich and Tyler 1999] to determine the successive contrast of the shown stimuli. 30 trials were used to measure the threshold at each point. Feedback was provided after each trial.

We displayed the previously described $256 \times 256$ pixels stimuli on an LCD computer screen running at $70 \mathrm{~Hz}$, with a pixel size of $0.295 \mathrm{~mm}$, positioned $2 \mathrm{~m}$ away from the subjects, thus ensuring a 120 pixels per degree raster resolution. The subjects looked at the stimuli with binocular vision and natural pupils. At each trial, the stimulus was presented during 5 seconds. During this interval, the contrast followed a Gaussian function of time with $\sigma=0.125 \mathrm{~s}$ and $\mu=2.5 s$. The stimulus therefore faded in, reached its maximum contrast after $2.5 \mathrm{~s}$, and then faded out. The inter-stimulus interval was set to $0.7 \mathrm{~s}$. 
The stimuli were presented at the center of the screen, in controlled lighting conditions. The screen luminance response was characterized before the experiment using a Konica Minolta LS-100 luminance meter, and the measured values were used to compute lookup tables mapping luminance values to RGB tristimulus values. The displayed images were grayscale, although we used the bit-stealing technique [Tyler 1992] to achieve a sufficient number of luminance levels. Four white L-shaped fixation marks were continuously presented at the corners of the stimulus display area during trials. Outside of this area, the screen remained uniformally gray with a luminance equal to the mean luminance of the stimuli $L_{0}=30.0 \mathrm{~cd} / \mathrm{m}^{2}$.

7 subjects (6 men and 1 woman) took part to the experiment. All were aged between 20 and 30 years, had a normal or corrected to normal vision, and did not report any difficulty about the experiment. One extra subject aborted the experiment before completion due to a technical problem; the incomplete data was ignored.

\subsubsection{Results}

Average threshold values for the $16\left(f_{0}, p\right)$ points of the Perlin noise parameter space are presented in Fig. 4a. The reported values are, as in ModelFest data [Watson 1999], estimates of the contrast value yielding $84 \%$ of correct answers. They are expressed in decibels $\left(1 d B=20 \log _{10}(c)\right.$, where $c$ is the Michelson contrast).

As could be expected, Perlin noise's base frequency parameter $\left(f_{0}\right)$ produces an effect analog to Gabor patches' spatial frequency. Qualitatively, the contrast sensitivity has the same behavior : low values for low frequency, a maximum for intermediate frequencies $\left(f_{0}=4 c p d\right.$ in our parameter space) and a decrease for higher frequencies. This behavior is present for all persistence values, although variations are less important at higher persistences. At any given frequency, sensitivity also decreases with persistence.

The descriptive statistics about our results confirm the good quality of our data. The variability among the observers $\left(R M S_{0}=\right.$ $2.73 d B)$, the error associated to the mean sensitivity of the observers $\left(R M S_{1}=1.59 \mathrm{~dB}\right)$, and the standard deviation of the observer sensitivities $\left(R M S_{2}=2.22 \mathrm{~dB}\right)$ are all inferior to the values reported by [Watson and Ahumada 2005] in their own dataset.

\subsection{Results discussion}

Comparison with ModelFest data describing contrast thresholds for Gabor patches (Fig. 4c) shows that sensitivity is globally lower for Perlin noise, except for high frequencies. This behavior can first be explained by the power spectrum of the stimuli : Gabor patches have much more compact power spectra, with all of the contrast energy tightly concentrated around the sine wave grating's spatial frequency. When this frequency corresponds to the peak sensitivity of the human visual system, very low thresholds are measured. Perlin noise textures have controlled power spectra too [Lagae et al. $2010]$ but the contrast energy is not as concentrated as for Gabor patches. Therefore, even when the power spectrum is centered on high sensitivity frequencies, much of the contrast energy is directed to less sensitive frequency channels, which gives higher contrast thresholds. This is even more the case for high persistence noise, where the multiple frequency bands that are added together to generate the final stimulus increase contrast energy in high frequencies, at the expense of high sensitivity medium frequencies.

We can also explain this behavior by the other specific features of Perlin noise textures : they are approximately isotropic, they are highly random (both statistically and visually), and they mimic natural textures. Current research shows that contrast sensitivity are

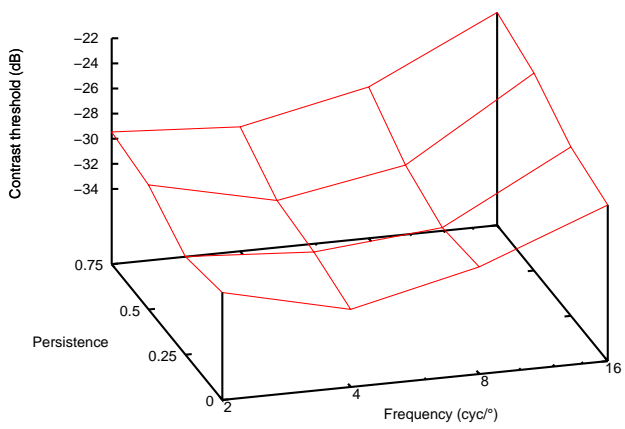

(a) Experimental values of mean contrast sensitivity for Perlin noise

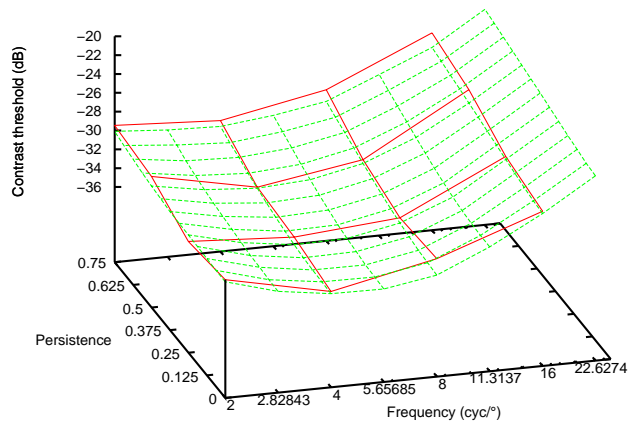

(b) Comparison between experimental values (in red) and model output (in green)

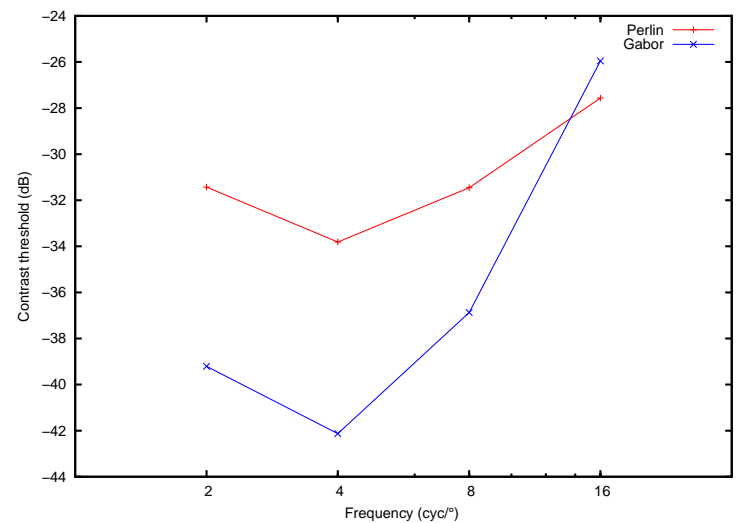

(c) Comparison of contrast sensitivities for Gabor patches (in blue ; data from the ModelFest project) and Perlin noise with $p=0$ (in red; our data).

Figure 4: Experimental results of Perlin noise contrast sensitivity assessment (Fig. 4a) compared to values predicted by a computational model (Fig. 4b) and to measured values for Gabor patches (Fig. 4c). 


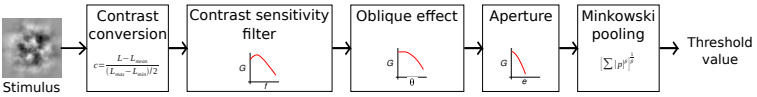

Figure 5: Schematic description of Watson \& Ahumada's "Standard A" model. See [Watson and Ahumada 2005] for details.

lower for isotropic and highly random stimuli like white noise [Watson and Ahumada 2005, stimulus 35] than for the non-random, highly anisotropic patterns usually used in contrast sensitivity research. The same can be said for natural images [Watson and Ahumada 2005, stimulus 43].

\subsubsection{Comparison and extension using Watson \& Ahumada's "Standard A" model}

Our bidimensional parameter space forced us to make some compromises when designing our experiment. In order to keep an acceptable experiment duration while maintaining a good data quality, we had to limit our exploration of the parameter space to 16 points, corresponding to only 4 values of each parameter.

In an attempt to extend this data and achieve a more complete exploration of the parameter space, we used a computational contrast sensitivity model. Contrast sensitivity models attempt to simulate the detection behavior of the human visual system and to predict contrast sensitivity thresholds for any stimulus.

[Watson and Ahumada 2005] describe several such models, fit their parameters using the ModelFest data base, discuss the results and propose two standard models combining relative simplicity and good threshold prediction. We chose to use one of these model, the "Standard A" model. It has a modular structure described in Fig. 5, and is composed of 5 components which implement different aspects of the behavior of the human visual system performing a detection task.

This model uses 7 parameters, which were estimated by the authors using numerical optimization routines to fit to the ModelFest data. We used the optimal model as described in the paper, without any modification, adaptation or parameter change. We implemented it as a Python program and ran it on our experimental stimuli and other Perlin noise textures.

We chose 128 points of the $\left(f_{0}, p\right)$ parameter space, with $f_{0}$ taking 8 values from 2 to $22.627 \mathrm{cpd}$ on a logarithmic scale, and $p$ taking 16 values from 0 to 0.9375 on a linear scale. 16 of these points correspond to our experimental stimuli, so we can directly compare the model output to the psychophysical data. For each point, 1000 Perlin noise stimuli were generated and observed by the model and the mean value was used.

Comparison between experimentally measured mean sensitivities values and values predicted by the model for these 16 points show an excellent fit between model and data : the RMS error is $R M S_{m}=0.892 d B$, which is inferior to the error [Watson and Ahumada 2005] found with the very dataset used to fit the model parameters $(1.329 d B)$. This result validates our experimental result by showing they are consistent with current knowledge of human contrast sensitivity. It also shows that the "Standard A" model is an efficient general model of human contrast detection, able to generalize to arbitrary stimuli with a good prediction power.

This good model fit for our stimuli allows us to use the model to estimate the human contrast sensitivities to Perlin noise textures with other parameters values, using the full 128 points dataset. The result is shown in Fig. 4b in green, together with the original experimental values in red. The extended values obtained using the model con-
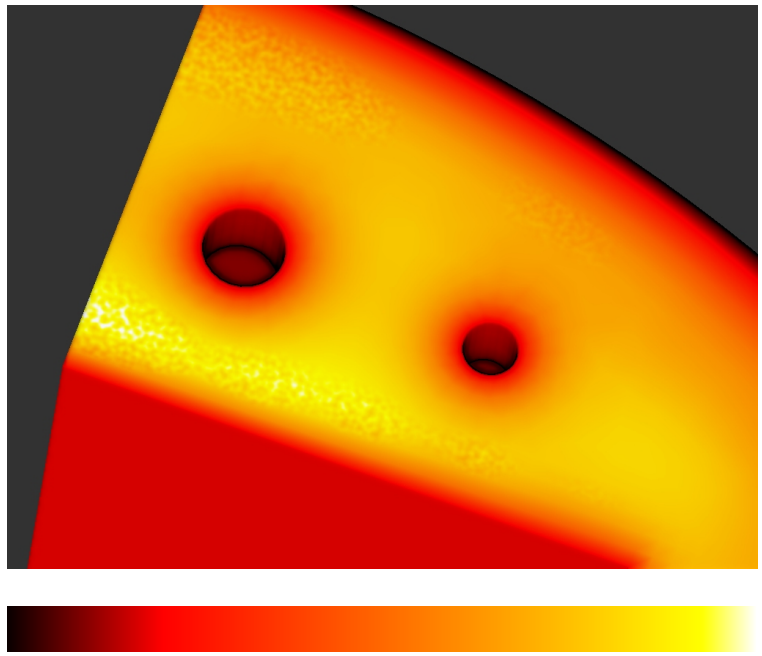

(a) Linear luminance black-body radiation color scale, piecewise-linear transfer function.

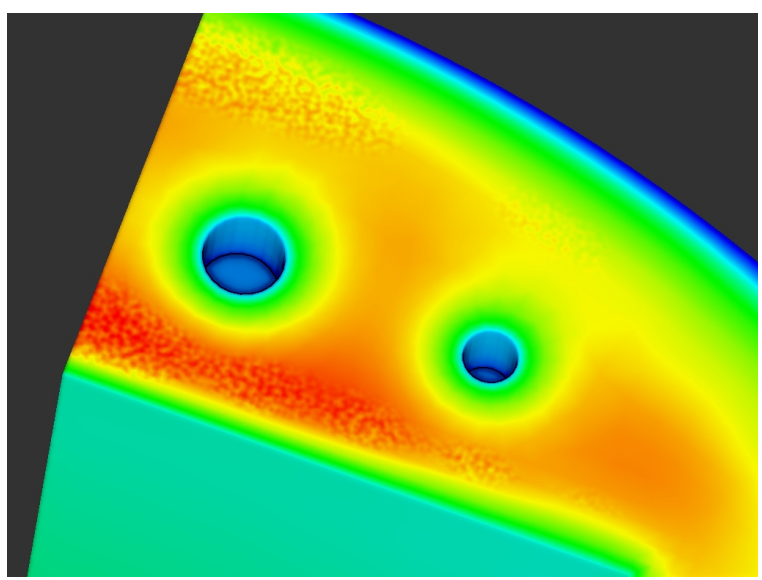

(b) Rainbow color scale, piecewise-linear transfer function.

Figure 6: Visualization of uncertain data from the same $3 D$ thermics simulation as presented in Fig. 3, using the non-linear transfer function shown on Fig. $3 f$ and two different color maps. The round, bubble-shaped high-uncertainty areas present in the data (see Fig. $3 b$ ) are highlighted by the chosen settings.

firm the precedent trends about the parameter effects : sensitivity always decreases with $p$, and reaches a maximum for intermediate $f_{0}$ values around $4-5 \mathrm{cpd}$.

\section{Perceptually adapted uncertainty visualiza- tion}

In this section we use the results of the constrast sensitivity experiment described in the previous section in order to drive the uncertainty visualization algorithm explained in section 2 .

As visualization scenes are very different and much more complex than Perlin noise experimental stimuli, we cannot expect our perceptual study to completely explain the perceptual behavior of the user. Especially, we measured thresholds for static noise while our visualization technique uses animated noise, we limited our study 
to foveal vision although visual exploration of large images uses both foveal and peripheral vision, and we used black and white luminance stimuli while our method is based on color maps, which implies both luminance and hue variations.

However, our study gives useful insights about the influence of base frequency and persistence on the visibility of the noise patterns. This allows us to build a perceptively adapted uncertainty visualization technique, using the Perlin noise parameters to control the uncertainty values that will be shown.

This process is illustrated in Fig. 7. While using a linear transfer function as explained in section 2.3, the contrast of the luminance variations introduced by the Perlin noise only depends on the uncertainty value and the color map. Thanks to our perceptual study, we can therefore alter the visibility of the Perlin noise patterns for a given uncertainty by adjusting $f_{0}$ and $p$ parameters, changing the visibility threshold value to make the contrast variations greater or lower than the threshold at a given point.

Since our experiment was conducted using luminance grayscale stimuli, special care must be taken when choosing the color map for our visualization. Among other benefits pointed by [Ware 1988], using a color map which presents a monotonic linear luminance progression make sure that the principles discussed above remain applicable at any point of the image. Fig. 6a shows an example using a linear black body radiation color map.

Other common color maps, like the ubiquitous "rainbow" color map, are less appropriate, but they usually have an approximately piecewise-linear luminance profile. Hence, even if we can't control the visibility of the uncertainty information for the entire image, the same technique can still be used locally to achieve perceptually adapted uncertainty visualization for one specific subset of the dataset. Figure $6 \mathrm{~b}$ shows such an example.

By combining this technique with the non-linear transfer function described in section 2.3 , we can both restrict the visualization to some interest values and control how and when these values are displayed. This allows us to easily and intuitively show some specific shapes or features of the uncertainty field, like the bubble-like high-uncertainty areas in Fig. 6.

\section{Conclusion and future work}

In this work, we have described a combination of algorithmic techniques and psychophysical study in order to visualize uncertain scalar information in complex 3D scenes. We built on existing colormap-based visualization tools and added noise patterns guided by the uncertainty information in an intuitive and non-disruptive way. We studied the noise visual primitives by a perceptual evaluation backed by a computational model, and integrated these works into a perceptually adapted uncertainty visualization method. In further work, we plan to extend our perceptual study to take into account the influence of color contrast and temporal variations, and to conduct user studies to confirm the usability of our technique for scientific and industrial data exploration. Furthermore we want to explore the use of more evolved noise patterns, in order to take into account uncertainty in a more complex way, closer to a complete probability density function (see [Luo et al. 2003]) instead of simple statistical values.

\section{Acknowledgements}

This research was funded by EDF R\&D and a CIFRE grant from Association Nationale de la Recherche et de la Technologie. We thank S. Ploix and C. Boucheny from EDF R\&D for their insights and fruitful discussion, and the subjects of our experiments for the time and attention they dedicated us.

\section{References}

Blakemore, C., ANd CAmpbell, F. 1969. On the existence of neurones in the human visual system selectively sensitive to the orientation and size of retinal images. Journal of Physiology 203, 237-260.

Campbell, F., And Robson, J. 1968. Application of Fourier analysis to the visibility of gratings. Journal of Physiology 197, 551-566.

Campbell, F., Kulikowski, J., And Levinson, J. 1966. The effect of orientation on the visual resolution of gratings. Journal of Physiology 187, 427-436.

Carney, T., Klein, S. A., Tyler, C. W., Silverstein, A. D., Beutter, B., Levi, D., Watson, A. B., Reeves, A. J., Norcia, A. M., Chen, C.-C., Makous, W., AND EcKSTEIN, M. P. 1999. The development of an image/threshold database for designing and testing human vision models. In Proc. SPIE, SPIE, 542-551.

Cedilnik, A., And Rheingans, P. 2000. Procedural annotation of uncertain information. In Proceedings Visualization 2000. VIS 2000 (Cat. No.00CH37145), IEEE, 77-84.

Duurcilov, S., Kim, K., Lermusiaux, P., and Pang, A. T. 2002. Visualizing scalar volumetric data with uncertainty. Computer \& Graphics 26, 239-248.

GRIEThe, H., AND Schumann, H. 2006. The Visualization of Uncertain Data: Methods and Problems. In Simulation und Visualisierung 2006 (SimVis 2006), SCS Publishing House e.V., T. Schulze, G. Horton, B. Prein, and S. Schlechtweg, Eds., vol. vi.

Gustavson, S., 2005. Simplex noise demystified. http: //webstaff.itn.liu.se/ stegu/simplexnoise/ simplexnoise.pdf.

Kao, D. L., Kramer, M. G., Luo, A., Dungan, J. L., And PANG, A. T. 2005. Visualizing distributions from multi-return lidar data to understand forest structure. The Cartographic Journal 42, 1, 35-47.

Kelly, D. H. 1979. Motion and vision II Stabilized spatiotemporal threshold surface. Journal of the Optical Society of America 69, 10 (Oct.), 1340.

Kelly, D. H. 1979. Motion and vision 1 Stabilized images of stationary gratings. Journal of the Optical Society of America 69, 9 (Sept.), 1266

KontSEvich, L. L., AND TYLER, C. W. 1999. Bayesian adaptive estimation of psychometric slope and threshold. Vision Research $39,2729-2737$.

Lagae, A., Lefebvre, S., Cook, R. L., Derose, T., Drettakis, G., Ebert, D. S., Lewis, J., Perlin, K., AND ZwICKER, M. 2010. State of the Art in Procedural Noise Functions. In EG 2010 - State of the Art Reports, Eurographics Association, E. Hauser and E. Reinhard, Eds.

LI, H., FU, C.-W., LI, Y., AND Hanson, A. J. 2007. Visualizing Large-Scale Uncertainty in Astrophysical Data. IEEE Transactions on Visualization and Computer Graphics 13, 6, 1640-1647.

Lundström, C., Ljung, P., Persson, A., And Ynnerman, A. 2007. Uncertainty visualization in medical volume rendering 
using probabilistic animation. IEEE Transactions on Visualization and Computer Graphics 13, 6, 1648-1655.

LuO, A., KAO, D., AND PANG, A. T. 2003. Visualizing spatial distribution data sets. In Proceedings of the symposium on Data visualisation 2003, Eurographics Association, 29-38.

Michelson, A. A. 1962. Studies in Optics. Univ. of Chicago Press.

MulLEN, K. T. 1985. The contrast sensitivity of human coulour vision to red-green and blue-yellow chromatic gratings. Journal of Physiology, 359, 381-400.

OWSLEY, C. 2003. Contrast sensitivity. Ophthalmology Clinics of North America 16, 2 (June), 171-177.

Pang, A. T., Wittenbrink, C. M., And Lodha, S. 1997. Approaches to uncertainty visualization. The Visual Computer $13,8,370-390$.

Perlin, K. 1985. An Image Synthetizer. In Proceedings of the 12th annual conference on Computer graphics and interactive techniques (SIGGRAPH '85), 287-296.

Perlin, K., 1999. Making Noise. http://www. noisemachine.com/talk1/.

Perlin, K. 2002. Improving Noise. In SIGGRAPH '02 Proceedings of the 29th annual conference on Computer graphics and interactive techniques, ACM New York, NY, USA, 681-682.

RIVEIRO, M. 2007. Evaluation of uncertainty visualization techniques for information fusion. In 10th International Conference on Information Fusion, IEEE, 1-8.

Robson, J., AND GRAHAm, N. 1981. Probability summation and regional variation in contrast sensitivity across the visual field. Vision Research 21, 409-418.

Skeels, M., Lee, B., Smith, G., and Robertson, G. G. 2009. Revealing uncertainty for information visualization. Information Visualization 9, 1 (May), 70-81.

TYLER, C. W. 1992. Bit stealing: how to get 1786 or more gray levels from an 8-bit color monitor. In Proceedings of SPIE, SPIE, 351-364.

VIARD, T. 2010. Algorithmes de visualisation des incertitudes en géomodélisation sur GPU. PhD thesis, Institut National Polytechnique de Lorraine.

WARE, C. 1988. Color Sequences for Univariate Maps: Theory, Experiments, and Principles. IEEE Computer Graphics And Applications Computer Graphics And Applications, September, 41-49.

Watson, A. B., And Ahumada, A. J. 2005. A standard model for foveal detection of spatial contrast. Journal of Vision 5, 717 740.

Watson, A. B., 1999. ModelFest web site. http://vision. arc.nasa.gov/modelfest/.

WATson, A. B. 2000. Visual detection of spatial contrast patterns: Evaluation of five simple models. Optics Express 6, 1 (Jan.), 12-33.

Wittenbrink, C. M., PAng, A. T., And Lodha, S. K. 1996. Craig M. Wittenbrink,. IEEE Transactions on Visualization and Computer Graphics 2, 3, 266-279.

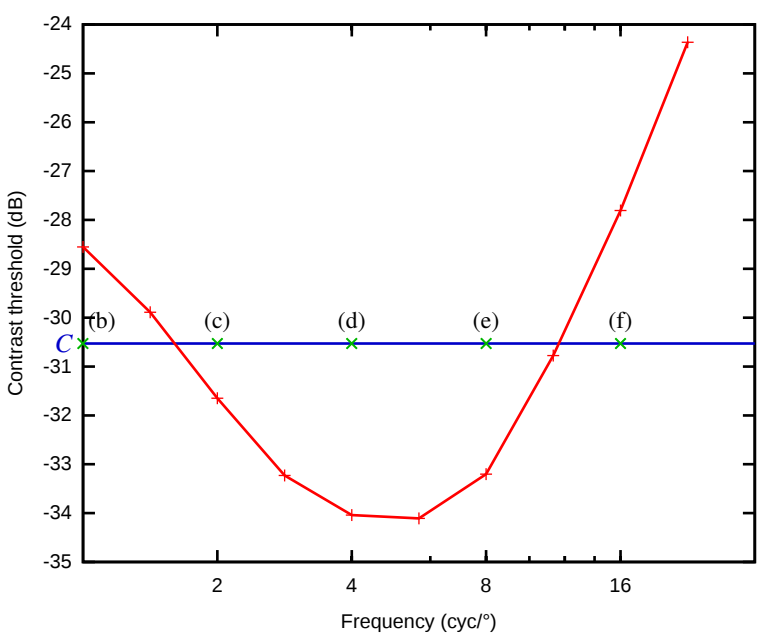

(a) Perlin noise contrast sensitivity

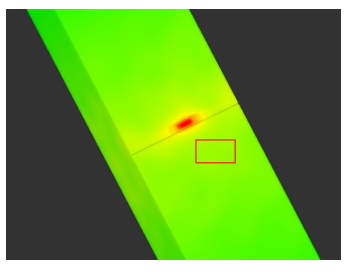

(b) $f_{0}=1 \mathrm{cpd}$

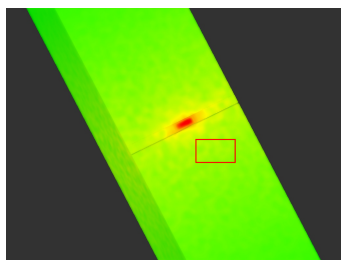

(d) $f_{0}=4 c p d$

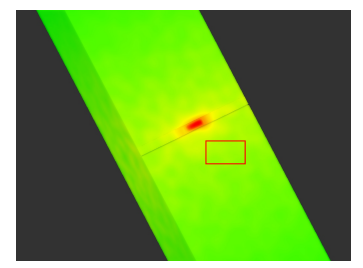

(c) $f_{0}=2 \mathrm{cpd}$

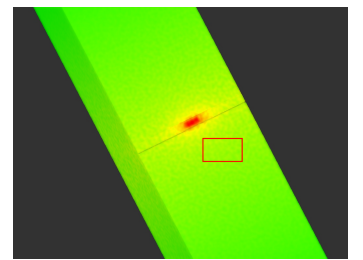

(e) $f_{0}=8 \mathrm{cpd}$

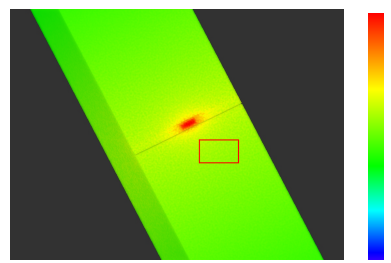

(f) $f_{0}=16 \mathrm{cpd}$

Figure 7: Effect of the $f_{0}$ parameter on the visibility of uncertainty information. A visualization of uncertain temperature data is shown for 5 different $f_{0}$ values (Fig. $7 b-7 f$ ), with $p=0$ and the same linear transfer function. Depending on the frequency value, the noise patterns showing uncertainty are above or below threshold sensitivity (Fig. 7a), and can be perceived or not in the red box. (Note: For best effect and correct spatial frequencies to be achieved on this figure, this page should be printed in A4 format on a high-definition color printer and viewed from a distance of 35 centimeters.) 